\title{
Synthesis and Properties of Oligomers Containing 1,4-Bis(thiophenene)buta-1,3-diyne and Bisphenol-A Derivatives
}

\author{
Jong Youn Bae, Jung Woo Park, Yong-Rok Kim," and Young Tae Park" \\ Department of Chemistrv, Keimyung University, Taegu 704-701, Korea \\ 'Deparment of Chemistr; Yonsei University, Seoul 120-749, Korea \\ Received August 13, 2001
}

Keywords : Oligomer, I,4-Bis(thiophene)buta-1,3-diyne. Bisphenol- $\Lambda$, $\Lambda$ bsorption. Fluorescence.

Conjugated polymers containing organic conjugate groups such as thiophene and acetylene have received considerable attention for the potential applications of electronic and optical materials in recent years. ${ }^{1-3}$ We have recently preparcd a new monomeric compound of 1,4-bis(5-chlorothiophenyl)buta-1,3-diyne, which contains organic conjugate groups such as both thiophene and diacetylene simultaneously. We have also reported the synthesis and properties of novel polycarbosilanes as well as polycarbogermanes containing 1,4-bis(thiophene)buta-1,3-diyne in the polymer main chain, ${ }^{4.5}$ and their excited-state energy dynamics. The polycarbosilanes and polycarbogermanes were prepared by the treatment of 1,4-bis(5-chlorothiophenyl)buta-1,3-diyne with n-butyllithium followed by co-polymerizations with dichlorodiorgano-silanes and dichlorodioraganogermanes, respectivcly.

We have also been interested in an aromatic nucleophilic substitution polymerization of 1.4-bis(5-chlorothiophenyl)buta-1,3-diyne. IJerein we describe the synthesis of new oligomeric materials containing 1,4-bis(thiophenc)buta-1,3diyne and bisphenol- $\Lambda$ derivatives in their main chains by nucleophilic substitution reaction along with the electronic and thermal properties of the oblained oligomers.

\section{Results and Discussion}

Synthesis of Oligomers. The monomer, 1,4-bis(5-chlorothiophenyl)buta-1,3-diyne (1) was prepared in a three-step procedure from commercially available 2-bromo-5-chlorothiophene according to the previously reported method (Scheme 1).'

The synthetic route for oligomers containing 1,4-bis(thiophenene)buta-1,3-diyne and bisphenol-A derivatives is outlined in Scheme 2. The copolymerization between 1 and bisphenol-A (2) was carried out in N-methyl-2-pyrrolidone (NMP)/toluene as solvent mixture in the presence of $\mathrm{K}_{2} \mathrm{CO}_{3}$

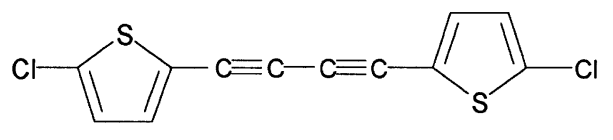

1

Scheme 1

"Corresponding author. E-mail: ytpark@kmu.ac.kr by a nucleophilic substitution reaction ${ }^{7.8}$ to afford the oligomer of poly|(1.4-bis(thiophene)buta-1,3-diyne)-co(bisphenol- $\Lambda$ )| (4). Toluene was used to remove the water generated upon heating to $110^{\circ} \mathrm{C}$ by azcolrope using a Dean-Stark trap. The material 4 was obtained in high yield as a black powder in the type of oligomer with a numberaverage molecular weight $\left(M_{n}\right)$ of $1.42 \times 10^{3}$ and a polydispersity $\left(M_{w} / M_{n}\right)$ of 1.80 . The oligomer of poly/(1.4bis(thiophene)buta-1,3-diyne)-co-(hexalluorobisphenol- $\Lambda$ )| (5) was also prepared in high yield following the same procedure for 4 using hexafluorobisphenol- $\Lambda$ (3) as comonomer. The obtained material 5 was found to be a black powder in the form of oligomer with $M_{11}$ of $1.44 \times 10^{3}$ and a polydispersity of 1.97 .

The obtained oligomeric materials have also been characterized by 'll NMR. IR speetra, as well as elemental analyses. The selected spectral data of oligomers 4 and 5 are summarized in Table 1 . In particular, the characteristic $C \equiv C$ stretching frequencies are observed at $2300 \mathrm{~cm}{ }^{\prime}$ in the IR spectra of 4 and 5 , indicating that the diacetylene groups remain intact during polymerization reactions. ${ }^{9}$ The clemental analyses data, which were included in the Experimental Section, were in good agreement with the proposed structure.

Properties of Oligomers. The oligomers 4 and 5 were soluble in common organic solvents such as TIJF and chloroform. The solubility of all the oligomers 4 and 5 allows us to perform studies on the properties in solution. The UV-vis absorption and the excitation spectra of $\mathbf{4}$ and 5 in THF were shown in Figures 1 and 2, respectively. The strong absorption bands with broad and weak tailing are observed in the absorption spectra of $\mathbf{4}$ and 5 (see the inset
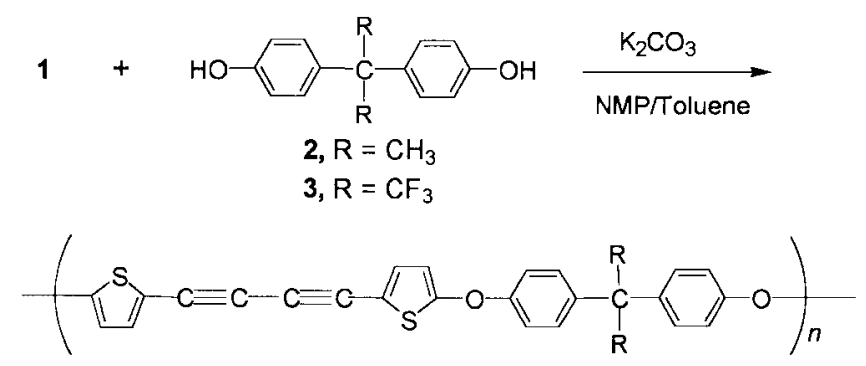

4, $\mathrm{R}=\mathrm{CH}_{3}(84 \%)$

5, $\mathrm{R}=\mathrm{CF}_{3}(89 \%)$

Scheme 2 
Table 1. Selected Properties of the Oligomers 4 and 5

\begin{tabular}{|c|c|c|c|c|c|c|}
\hline Oligomer & $M_{i j}^{\sigma}$ & $M H_{W} M_{i j}^{\sigma}$ & $\begin{array}{l}{ }^{1} \mathrm{H} \text { NMR } \\
\delta \text { (ppnn) }\end{array}$ & $\begin{array}{c}\text { IR } \\
v_{(\mathrm{C}=\mathrm{C})}\left(\mathrm{cm}^{-1}\right) \\
\end{array}$ & $\begin{array}{c}\text { Fluorescence } \\
\lambda_{\max }(\mathrm{nm})\end{array}$ & $\mathrm{TGA}^{\circ}$ \\
\hline 4 & $1.42 \times 10^{5}$ & 1.80 & $\begin{array}{l}1.40-1.60(\mathrm{bs}) \\
6.50-7.60(\mathrm{~m})\end{array}$ & 2300 & 460 & 76 \\
\hline 5 & $1.44 \times 10^{3}$ & 1.97 & $6.60-7.60(\mathrm{~m})$ & 2300 & 465 & 84 \\
\hline
\end{tabular}

"Determined by GPC in THF relatice to polystyrene standards. ${ }^{b}$ In THF. ${ }^{C}$ Excitation wavelength of $360 \mathrm{nmm} .{ }^{d_{0}} \mathrm{o}$ Weight remaining at $400{ }^{\circ} \mathrm{C}$ in nitrogen.

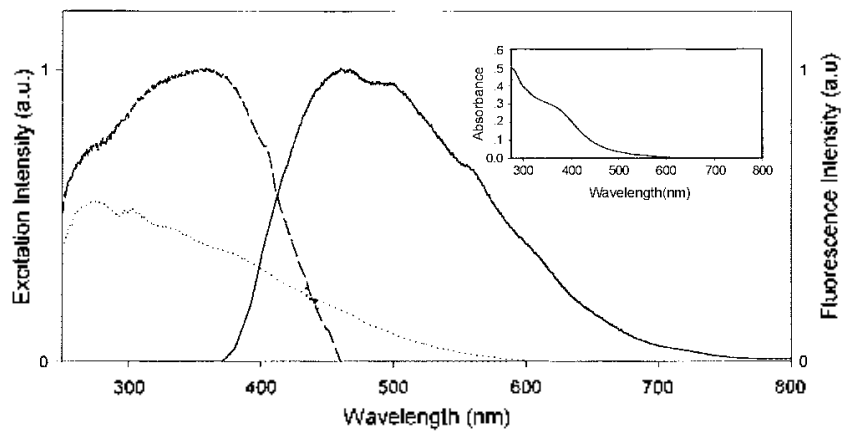

Figure 1. Fluorescence spectrum at 360 nun excitation (solid), excitation spectra at $460 \mathrm{~nm}$ of detection (short dash), and at 600 nm of detection (dotted) of 4 in THF. Inset is the absontion spectrum of 4 in THF.

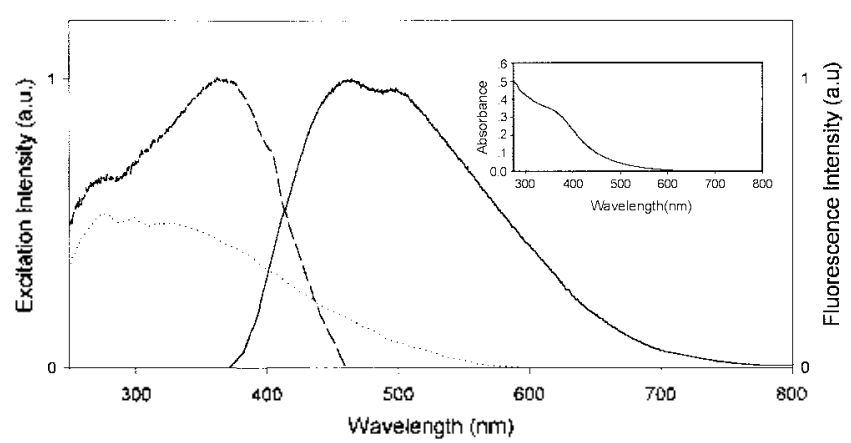

Figure 2. Fluorescence spectrum at 360 nun excitation (solid), excitation spectra at $460 \mathrm{~mm}$ of detection (short dash), and at 600 $1 \mathrm{~mm}$ of detection (dotted) of $5 \mathrm{in}$ THF. Inset is the absorption spectrum of 5 in THF.

spectra in Figures 1 and 2. respectively). The excitation spectra for 4 and 5 at the detection wavelength of $460 \mathrm{~nm}$ show the strong excitation bands at the $\lambda_{\text {max }}$ of 361 and 371 nu. respectively. The broad and weak excitation bands are also observed at the detection wavelength of $600 \mathrm{~nm}$ for both 4 and 5 . These strong absorption and excitation bands may be attributed to the chromophores such as thiophene. diacetylene. bisphenol-A derivatives. and partially delocalized $\pi$-conjugate groups along the backbone. The timeresolved spectroscopic experiments are being in progress to understand the origins of these excitation bands.

The fluorescence emission spectra of 4 and 5 at the excitation wavelength of $360 \mathrm{~nm}$ in THF were also shown in Figures 1 and 2. respectively. The fluorescence spectra of 4 and 5 indicate the strong emission peaks at the $\lambda_{\text {max }}$ of 460 num with the emission band of $374-760 \mathrm{~nm}$ and at the $\lambda_{\text {max }}$ of $465 \mathrm{~nm}$ with the emission band of $375-760 \mathrm{~nm}$. respectively.
These strong emission peaks are due to the fluorophores of $\pi$-conjugated system in the oligomers 4 and 5 . which contain the partially delocalized $\pi$-conjugate group along the main chain

Although the origins of such broad fluorescence spectra are not clear at this moment. the observed broadness of the emission spectra includes an important application respect in electro-optic field as the white light continuum generation that is not usual property especially for the low molecular weight oligomers. Such phenomenon usually depends on the several factors which can be the inhomogenieties in the number of repeating units of the prepared oligomers, the electronic energy dynamics of $\pi$-conjugation along the backbone. and the geometrical molecular structures in their electronic ground and/or excited states. The time resolved fluorescence spectroscopic studies on these factors are also in progress.

The thermal stabilities of oligomers $\mathbf{4}$ and 5 in nitrogen atmosphere were determined by themogravimetric analysis (TGA). Oligomer 4 is thermally stable up to $150^{\circ} \mathrm{C}$. Rapid weight loss of $33 \%$ occurs between 260 and $6500^{\circ} \mathrm{C}$. When 4 is heated to $900^{\circ} \mathrm{C}$ by the heating rate of $10^{\circ} \mathrm{C} / \mathrm{min}$, a residue of $50 \%$ remains (see the broken line in Figure 3 ). Oligomer $\mathbf{5}$ is themally stable to $200^{\circ} \mathrm{C}$. Rapid weight loss of $36 \%$ occurs between 300 and $600^{\circ} \mathrm{C}$. At $900^{\circ} \mathrm{C}$. $48 \%$ of the initial sample weight of 5 remains (see the solid line in Figure 3).

On the differential scanning calorimetry (DSC) curves of the obtained oligomers, the anticipated exothemic peaks due to the cross-linking of diacetylene group were not observed. while the exothermic ones were reported in the

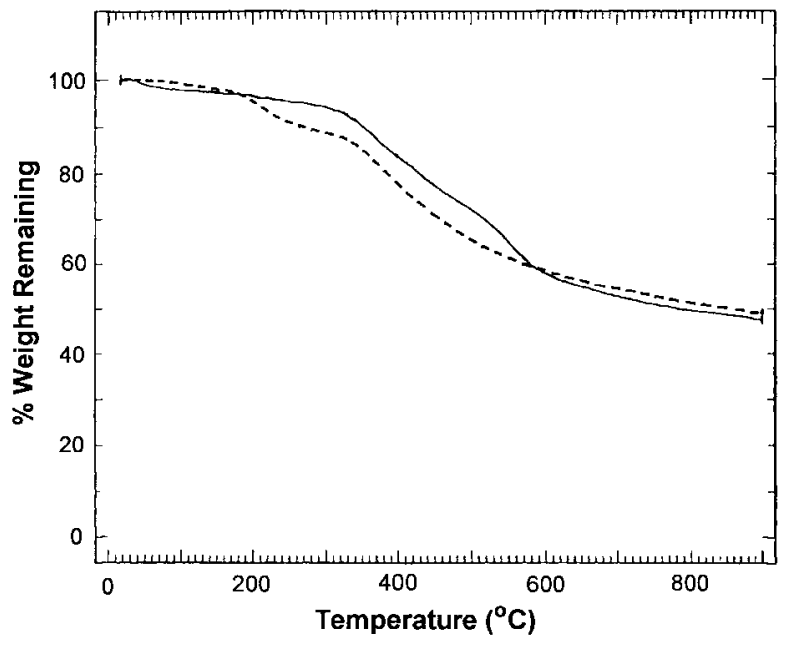

Figure 3. TGA thennograms of 4 (---) and 5 (- 
diacetylene-containing organic polymers such as poly(2.5diethyny lthiophene) ${ }^{11}$ as well as the copolymer of 1.4-bis(4fluorophenyl)buta-1.3-diyne and hexafluorobisphenol- $\mathrm{A}^{7}$

In conclusion, we have successively prepared the oligomeric materials which contain 1,4-bis(thiophene)buta-1,3diyne and bisphenol-A derivatives in the main chain. All of these materials are soluble in THF as well as chloroform. and are thermally stable up to $150{ }^{\circ} \mathrm{C}$ under nitrogen. The UV-vis absorption and the excitation spectra exhibit the strong absorption bands with broad and weak tailing. The fluorescence spectra show the broad emission bands of 374 . $760 \mathrm{~nm}$ with the enission $\lambda_{\text {max }}$ values in the range of 460 $465 \mathrm{~lm}$. Both the strong absorption and emission spectra indicate that the obtained oligomers contain the conjugate groups such as thiophene. diacetylene, and bisphenol-A derivatives as well as partially delocalized $\pi$-conjugate groups in the their main chain. Furthermore. both the strong absorption and emission spectra suggest the application possibility of these molecular system s as strong white light emission mediums.

\section{Experimental Section}

General Procedures. All chenicals were purchased from Aldrich Chemicals Co. Solvents were purified by conventional methods and were freshly distilled under argon prior to use. All reactions were carried out under argon atmosphere. ${ }^{1} \mathrm{H}$ and ${ }^{13} \mathrm{C}$ NMR spectra were recorded on either a Bruker ARX 300 or a JEOL JNM-EX90A spectrometer. Chenical shifts were measured using tetramethylsilane or the solvent as intental standards. IR spectra were recorded with either a Bruker IFS-48 or a Shinadzu IR- 470 spectrometer. UV-vis spectra were run on a Hewlett Packard 8452A spectrophotometer. Fluorescence emission spectra were obtained on a Hitachi F- 4500 . Gel permeation cluronatography (GPC) analyses were performed on a Waters model 510 system with a Waters Styragel HR 3 column and refractive index detector at $25^{\circ} \mathrm{C}$. The eluent was $\mathrm{THF}$ at a flow rate of $1.0 \mathrm{~mL} / \mathrm{min}$. The calibration was made with a series of monodispersed polystyrene standards. Themogravintetric analyses (TGA) were performed on a Shiniadzu TGA-50 instrument. The temperature was increased at the rate of $10^{\circ} \mathrm{C} / \mathrm{min}$ from 25 to $900^{\circ} \mathrm{C}$ with nitrogen flow rate of $20 \mathrm{~mL} / \mathrm{min} . T_{\mathrm{g}}$ was measured by differential scanning calorimetry in nitrogen on a Perkin-Elmer DSC-7. Elemental analyses were performed by a Fisons EA 1108 elemental analyzer.

1,4-Bis(5-chlorothiophenyl)buta-1,3-diyne (1) was prepared from 2-bromo-5-chlorothiophene according to the previously reported method. ${ }^{4}$

Copolymerization of Monomer 1 and Bisphenol-A (2). In a $250 \mathrm{~mL}$ three-necked round botton flask equipped with an argon inlet, stirring bar. Dean-Stark trap. and condenser. 1 (1.50 g. $5.3 \mathrm{mmol}), 2(1.21 \mathrm{~g} .5 .30 \mathrm{mmol})$ and NMP $(60 \mathrm{~mL})$ were placed under argon atmosphere. $\mathrm{K}_{3} \mathrm{CO}_{3}(1.10 \mathrm{~g} .7 .95$ mmol) and $40 \mathrm{~mL}$ of toluene were then added. The reaction misture was heated until the toluene began to reflux. Water formed during the reaction was removed as an azeoptrope with toluene over $4.5 \mathrm{~h}$ periods. The reaction mixture was slowly raised to $180-190^{\circ} \mathrm{C}$ for $2.3 \mathrm{~h}$. The polymer was isolated by precipitating into $100 \mathrm{~mL}$ of methanol. and washed with water/methanol 4-5 times to remove excess salts. The suspension was warmed with water/methanol, filtered. and dried in a vacuum oven at room temperature. Poly[(1.4-bis(thiophene)buta-1.3-diyne)-co-(bisphenol-A)] (4), $1.91 \mathrm{~g} .84 \%$ was obtained as a black powder with $2.560 /$

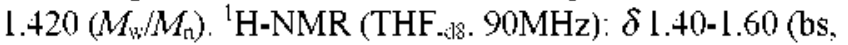
$6 \mathrm{H}$ ). $6.50-7.60$ (m. 12H); IR (KBr pallet): $v 3350,3100$. 2960. 2300, 1660, 1600. 1500, 1410, 1380. 1360, 1215. 1170, $1050,830,700 \mathrm{~cm}^{-1}$. Anal. Calcd for $\left(\mathrm{C}_{27} \mathrm{H}_{18} \mathrm{O}_{2} \mathrm{~S}_{2}\right)_{\mathrm{n}}$ C. 73.94: H, 4.14, S; 14.62. Found: C. 71.88; H. 4.22, S: 14.99. UV-ris absorption, excitation, and fluorescence spectra of 4 were measured in diluted THF solution $\left(\cong 1.0 \times 10^{-6} \mathrm{M}\right)$ and described in the Results and Discussion. as well as TGA thermogram.

Copolymerization of Monomer 1 and Hexafluorobisphenol-A (3) was carried out following the identical manner used in the preparation of 4 . using $1(1.50 \mathrm{~g} .5 .30 \mathrm{mmol})$ and 3 (1.78 g. $5.30 \mathrm{mmol}$ ). Poly[(1.4-bis(thiophene)buta-1.3diyne)-co-(hexafluorobisphenol-A)] (5), $2.56 \mathrm{~g} .88 \%$ was obtained as a black powder with $2,830 / 1.440\left(M_{\mathrm{w}} / M_{\mathrm{n}}\right) .{ }^{1} \mathrm{H}-$ NMR (THF.d\&, $90 \mathrm{MHz}$ ): $\delta 6.60-7.60(\mathrm{~m}, 12 \mathrm{H})$; IR (KBr pallet): $v 3350,3100.2960 .2300,1700,1660.1605 .1505$, 1435. 1370, 1260, 1240, 1170, 1130. 960,830. $700 \mathrm{~cm}^{-1}$; Anal. Calcd for $\left(\mathrm{C}_{27} \mathrm{H}_{12} \mathrm{~F}_{6} \mathrm{O}_{2} \mathrm{~S}_{2}\right)_{n}: \mathrm{C} .59 .34 ; \mathrm{H}, 2.21, \mathrm{~S} ; 11.73$. Found: $\mathrm{C}, 59.46 ; \mathrm{H} .2 .34, \mathrm{~S} ; 11.14$. UV-vis absorption. excitation. and fluorescence spectra of 5 were measured in diluted THF solution ( $\cong 1.0 \times 10^{-6} \mathrm{M}$ ) and described in the Results and Discussion along with TGA themogram.

Acknowledgment. This work was partially supported by the Korea Science and Engineering Foundation (Project No. KOSEF 2001-1-12200-002-2). Y.-R. Kim thanks for the funancial support from CRM-KOSEF grant (1998G0102).

\section{References}

1. Tour. J. M. Acc. Chem. Res. $2000,33.791$.

2. Handbook of Organic Conductive Molecules and Polymers: Nalwa. H. S., Ed.: John Wiley \& Sons: Chichester, England. 1997.

3. Coningated Pohners and Related Haterials: The Intercomection of Chentical and Electronic Strncture: Salaneck. W. R.. Lundström. I.. Rảnby: B.. Eds.: Oxford University Press: Osford. England. 1993.

4. Seo. I. K.: Park, Y. T.: Kim. Y.-R. Bull Korean Chem. Soc. 1999. 2016). 677 .

5. Bae. I. Y.: Kiml. Y.R. Park. Y. T. Bull Korean Chent Soc. 2000. $21(8) .831$.

6. Hwang. I.-W.: Song. N. W.: Kim. D.: Park. Y. T.: Kim. Y.-R. J. Polvm. Sci. Part B: Polm. Phys. 1999.37.2901.

7. Park. Y. T: Chiesel, N.: Economy. J. Hol Coyst Liq. Crust 1994. 247.351.

8. Gilchrist. T. L. Heterocyclic Chemism: 2nd ed.: Longman Scientific \& Technical: Essex. England. 1992: p 218.

9. Pretsch. E.: Bühlmann. P.: Affolter. C. Structure Detenmination of Organic Compounds. Tables of Spectral Data, 3rd ed: SpringerVerlag: Berlin. 2000.

10. Park. Y. T: Seo. I. K.: Kim. Y.-R. Bull. Korean Chem. Soc. 1996. $17(5) .480$. 